\title{
Improvements of Liver MR Imaging Clinical Protocols to Simultaneously Quantify Steatosis and Iron Overload
}

\author{
R. Ternifi ${ }^{a}$, P. Pouletaut ${ }^{a}$, M. Sasso ${ }^{\text {b }}$, V. Miette $^{\text {b }}$, F. Charleux ${ }^{\text {c }}$, S.F. Bensamoun ${ }^{\text {a,* }}$ \\ ${ }^{a}$ Sorbonne University, Université de technologie de Compiègne CNRS, UMR 7338 Biomechanics and Bioengineering, Centre de Recherche de Royallieu, \\ Compiègne, France \\ ${ }^{\mathrm{b}}$ Echosens, Département R\&D, Paris, France \\ ${ }^{\mathrm{c}}$ ACRIM-Polyclinique Saint Côme, Compiègne, France
}

Received 12 January 2018; accepted 19 April 2018

Available online 7 May 2018

\section{Highlights}

- Existing clinical protocols could be improved to provide simultaneous quantification of iron and fat.

- Advanced liver protocols can now provide $2 \mathrm{D}$ or volume cartographies of iron and fat.

- Estimation of the limits of the liver MRI methods.

- Comparison of clinical MRI protocols for iron and fat quantification.

\section{Graphical abstract}

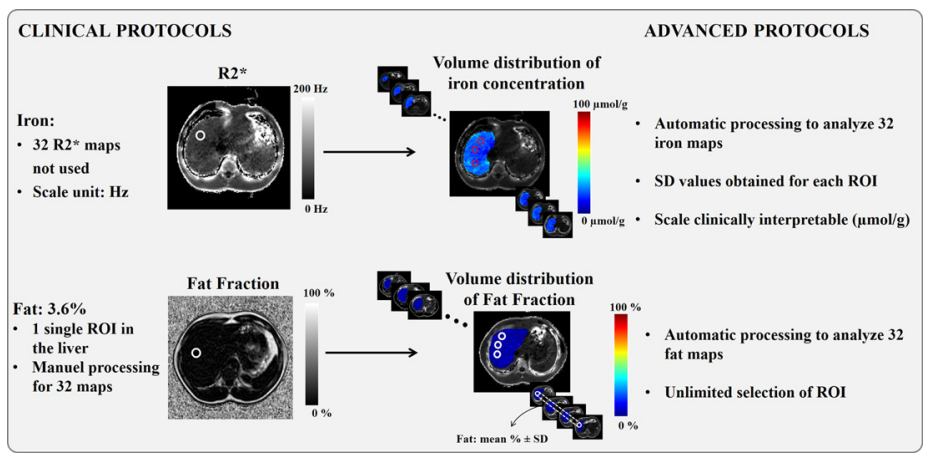

\section{Abstract}

Purpose: Fat accumulation and iron overload are important cofactors in chronic liver disease. Clinical quantifications of fat fraction and iron are currently assessed using MRI protocols. The purpose is to improve these measurements to simultaneously provide iron and fat maps from a single acquisition.

Methods: Ten healthy volunteers and ten patients with steatosis underwent MRI for fat fraction (FF: IDEAL-IQ ${ }^{\circledR}$ ), iron overload concentration (IOC: Gandon, Starmap ${ }^{\circledR}$ ) and viscoelastic characterization (MR-Touch ${ }^{\circledR}$ ). IDEAL-IQ ${ }^{\circledR}$ data, the clinical FF reference, were compared to the advanced Gandon protocol, post-treated with a 3 pt Dixon method. The originality was to use IDEAL-IQ ${ }^{\circledR}$ fat sequence to quantify iron volumetrically using the Wood equation. To validate the iron data, the reference Gandon protocol was applied and improved to provide map of IOC. Then, IOC data were also compared to another clinical sequence (Starmap ${ }^{\circledR}$ ) which was also improved (scale, number of ROI). The estimated error associated with each method was evaluated with the coefficient of variation.

Results: IDEAL-IQ ${ }^{\circledR}$ and Gandon protocols were modified to provide simultaneously FF and IOC maps (2D, volume). Healthy FF were in the same range with all protocols $(\approx 3 \%)$. For patients with steatosis, Gandon protocols underestimated the FF value $(\approx 7 \%)$ compared to IDEAL-IQ ${ }^{\circledR}$.

\footnotetext{
* Corresponding author at: Université de Technologie de Compiègne (UTC), Laboratoire de BioMécanique et BioIngénierie, UMR CNRS 7338, CS 60319, 60203 Compiègne Cedex, France.

E-mail address: sabine.bensamoun@utc.fr (S.F. Bensamoun).
} 
Healthy and fibrosis patients were correctly diagnosed (no hemochromatosis) with all the protocols and viscoelastic properties were in the same range.

Conclusion: Manufacturer's tools were improved to simultaneously quantify liver markers saving time for the patient and the clinical setting. These parameters are of great value for clinical diagnostics and novel therapeutics to treat liver diseases.

(c) 2018 AGBM. Published by Elsevier Masson SAS. All rights reserved.

Keywords: Liver MRI/MRE; Clinical protocols; Fat fraction; Iron overload

\section{Introduction}

Steatosis and hemochromatosis are common histological features in the general population and in patients with chronic liver disease. They can result from several causes, such as high alcohol consumption, chronic viral hepatitis or metabolic dysfunction [1,2]. Intracellular fat accumulation and iron overload are commonly regarded as two important cofactors in liver disease that can lead to fibrosis and cirrhosis [2]. Non-targeted percutaneous liver biopsy with direct histological visualization is the current gold standard to diagnose hepatic steatosis and iron load [3].

Magnetic resonance imaging (MRI) sequences have been proposed for non-invasive detection and quantification of liver fat and iron content. Most MR protocols have been correlated with biopsy to validate the method and are now used in clinical routines.

For iron overload quantification, the well-known Gandon's method is commonly applied [3]. This protocol is composed of five MR sequences (different gradient echo GRE with fixed TR and variable TE and flip angle) using the liver-to-muscle signal intensity ratio as a marker of liver iron concentration for a local region. Subsequently, Gandon's protocol has been improved with the measurement of additional signals (background, spleen) and the acquisition of one T2* MR sequence $[4,5]$ to measure the $\mathrm{T} 2 *$ relaxation time that is strongly correlated with iron concentration. Gandon's measurements are based on data extracted from an average value of three liver regions of interest (ROI). However, the iron standard deviation is typically high [4] which complicates interpretation. In addition to Gandon's protocol, imaging companies have also implemented iron-specific protocols such as the Starmap ${ }^{\circledR}$ sequence [6] from General Electric. This sequence is based on standard multi-echo gradient (GRE) and has been validated with liver biopsy. Starmap ${ }^{\circledR}$ provides a spatial distribution of iron represented by a scale unit in $\mathrm{Hz} / \mathrm{ms}$, which is also difficult to interpret clinically. It has been demonstrated that later echoes of the Starmap ${ }^{\circledR}$ sequence show a more rapid signal decline for tissue rich in iron than for health tissue [6].

To estimate fat fraction, Gandon's protocol, based on a Dixon acquisition with $\mathrm{T} 2 *$ correction method, is also used. From three liver ROIs, the fat data is provided in percentages without standard deviation. Similar to iron quantification, imaging companies have proposed fat-specific sequences such as IDEAL-IQ $^{\circledR}$ (Iterative Decomposition of water and fat with Echo Asymmetry and Least squares estimation) $[7,8]$ from General Electric. IDEAL-IQ relies on quantitative chemical shift-based water-fat separation to provide a map of fat fraction, but only a single ROI may be placed inside the liver.

The study objective was to improve the existing MRI protocols (Gandon, Starmap ${ }^{\circledR}$, IDEAL-IQ ${ }^{\circledR}$ ) to simultaneously provide iron and fat cartographies from a single acquisition. These spatial distributions, either 2D or volume, may contribute to improve follow-up and diagnosis of liver fibrosis. In addition, MR elastography was performed to characterize the stiffness of the liver. The development of non-invasive and quantitative MR imaging markers (iron overload, fat fraction, and viscoelastic parameters) are of great value for clinical diagnostics and novel therapeutics to treat liver diseases.

\section{Patients and methods}

\subsection{Study population}

Twenty subjects comprised of ten healthy volunteers ( 7 men, 3 women, age range: $21-70$ years, mean age $=43 \pm 18$ years, mean BMI (body mass index) $=26.13 \pm 3.6 \mathrm{~kg} / \mathrm{m}^{2}$ ) without liver damage, and ten nonalcoholic patients $(7 \mathrm{men}, 3$ women, age range: $46-78$ years, mean age $=59.6 \pm 8$ years, mean $\mathrm{BMI}=30.2 \pm 3.4 \mathrm{~kg} / \mathrm{m}^{2}$ ) with steatosis (level of fat between $5 \%$ and $31 \%$ ) were recruited from the gastroenterology department at the Saint Côme polyclinic (Compiègne, France). Each subject underwent MR imaging tests for fat quantification, iron overload, and MR elastography tests for viscoelastic characterization. Total elapsed time for all tests was $10 \mathrm{~min}-$ utes. Exclusion criteria were claustrophobia, mental instability, or presence of hepatitis. This study was approved by the Institutional Review Board of Amiens Hospital. All subjects had the experimental protocol explained and gave their informed written consent prior to admission into the study.

\subsection{MRI protocols for fat quantification}

The subjects lay supine in a $1.5 \mathrm{~T}$ Signa HDx MRI machine (GE, Milwaukee, WI) with an abdominal coil for the MRI acquisitions. Fat quantification is clinically obtained by applying the standard IDEAL-IQ ${ }^{\circledR}$ (Fig. 1A) sequence, implemented by General Electric. IDEAL-IQ ${ }^{\circledR}$ is a gradient echo sequence $\left(\mathrm{TR}=11.5 \mathrm{~ms}, \mathrm{FA}=7^{\circ}\right)$ applying $6 \mathrm{TEs}$ from $2.4 \mathrm{~ms}$ to $9.6 \mathrm{~ms}$. The following parameters were applied: receiver bandwidth $=166.7 \mathrm{kHz}, \mathrm{FOV}=26-33 \mathrm{~cm}$, matrix $=160 \times 160$, slice thickness $=10 \mathrm{~mm}, 24$ slices covering $24 \mathrm{~cm}$ in the superior inferior direction. The scan time was approximately $21 \mathrm{~s}$ with a single breath-hold. For this study, 32 fat fraction 


\section{List of abbreviations}

$\begin{array}{llll}\text { MRI } & \text { magnetic resonance imaging } & \text { IOC } & \text { iron overload concentration } \\ \text { MRE } & \text { magnetic resonance elastography } & \text { FF } & \text { fat fraction } \\ \text { GRE } & \text { gradient echo } & \text { FOV } & \text { field of view } \\ \text { ROIs } & \text { regions of interest } & \text { MMDI } & \text { multimodel direct inversion } \\ \text { SI } & \text { signal intensity } & \text { SD } & \text { standard deviation }\end{array}$
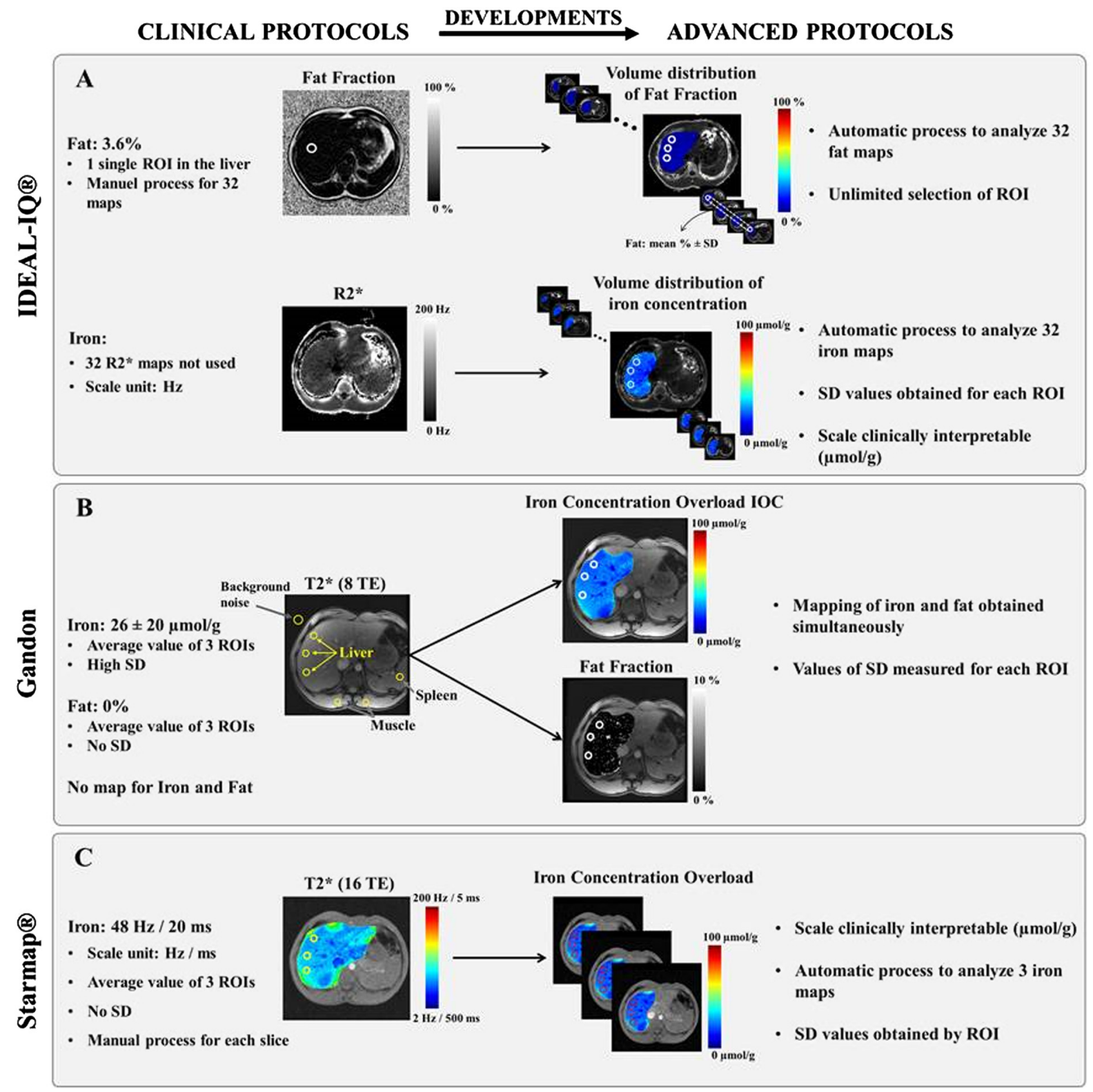

Fig. 1. Summary of the different clinical and advanced protocols to quantify fat fraction and iron overload concentration. (For interpretation of the colors in the figure(s), the reader is referred to the web version of this article.)

(FF) maps were reconstructed from the calculated water-only and fat-only maps $(\mathrm{FF}=\mathrm{fat} /($ water + fat $) \times 100)($ Fig. $1 \mathrm{~A})$. Data have been provided as percentages with standard deviation from prescribed ROIs, limited to three per slice within the liver, spleen and fat, respectively. To increase the number of ROIs and to quantify the FF over the entire liver an auto- mated method has been developed using Image J [9] 1.46/Java 8 software (National Institute of Health, Bethesda, MD, United States).

Another way to obtain the spatial distribution of the fat has been to improve the Gandon protocol (Fig. 1B) usually used for iron quantification. A 3pt Dixon method was applied to the T2* 
acquisitions. The method is based on the mean signal intensity (SI) in each ROI measured at the second opposed-phase (OP) time echo and two consecutive, in-phase (IP1, IP2) echo times that occurred before and after the OP times [10,11]. Fat fraction (FF) was then calculated for each pixel with the following equation: $\mathrm{FF}=100 \times($ SImean_IP-SI_OP $) /(2 \times$ SImean_IP $)$ where SImean_IP $=($ SI_IP1 + SI_IP2)/2 $($ Fig. 1B $)$.

\subsection{MRI protocols for iron overload quantification}

The originality of the present study has been to use the IDEAL-IQ ${ }^{\circledR}$ fat sequence to quantify iron volumetrically through the $32 \mathrm{R} 2 *$ liver slices which are not used in clinical practice (Fig. 1A). The advanced IDEAL-IQ ${ }^{\circledR}$ protocol used the $32 \mathrm{R} 2 *(1 / \mathrm{T} 2 *)$ liver slices, recorded with a scale unit in $\mathrm{Hz}$, with the Wood equation applied to each pixel. This advanced IDEAL-IQ protocol thus provides a volume representation of IOC values in $\mu \mathrm{mol} / \mathrm{g}$. To validate the iron data, a well known protocol, called Gandon (Fig. 1B) was applied with a single breath-hold gradient echo (GRE) sequence (TR $=120 \mathrm{~ms}$, FA $=20^{\circ}$ ) composed of $8 \mathrm{TEs}$, in multiples of $2.4 \mathrm{~ms}$, to acquire one T2* liver slice. From this acquisition, seven regions of interest (ROIs) (120 pixels, about $150 \mathrm{~mm}^{2}$ per ROI) were manually placed within the $\mathrm{T} 2 *$ acquisition: three within the liver, inside segments VII (posterior superior area) and VIII (anterior superior area), one inside the spleen, two others in the right and left paraspinal muscles, and one in the image background. The IOC value was calculated using the IronByMR [12] software, which is a plug-in developed in Java and integrated into the ImageJ [9] software package (NIH, Bethesda, USA). IronByMR estimates liver iron overload by comparing the $\mathrm{T} 2 *$ signal liver-to-muscle intensity ratio against background noise. Using Wood's equation [6]: $[\mathrm{Fe}]=0.4535 / \mathrm{T} 2 *+3.607$, the IOC value $(\mu \mathrm{mol} / \mathrm{g})$, along with standard deviation, can be calculated from the measured $\mathrm{T} 2 *$ value (s). These data, however, give only a global estimation (mean of three ROIs) of the iron over-

\section{Complex shear modulus}

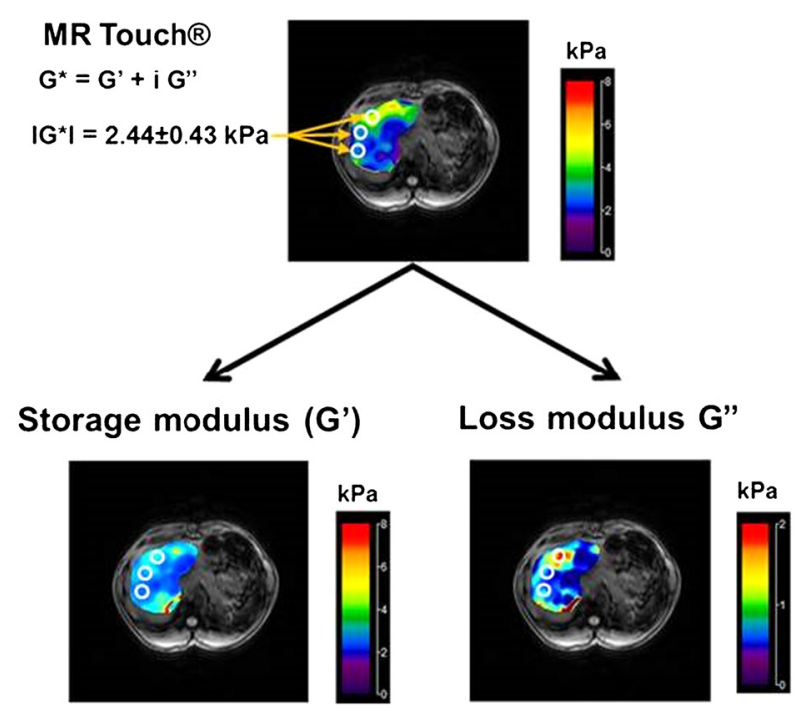

Fig. 2. Viscoelastic properties of the liver. load. An improvement of Gandon's protocol, called "Advanced Gandon", was developed to provide a pixel-wise map of IOC (Fig. 1B). Here T2* values for each pixel are deduced from an exponential fitting curve with a constant offset of background.

Another way to compare the IOC values, obtained with IDEAL-IQ ${ }^{\circledR}$ fat sequence, has been to use an iron protocol implemented by General Electric and called Starmap ${ }^{\circledR}$ (Fig. 1C). It is based on a GRE sequence [6] $\left(\mathrm{TR}=52.8 \mathrm{~ms}, \mathrm{FA}=25^{\circ}\right.$, FOV $=42-42 \mathrm{~cm}$, matrix $=512 \times 512$ ) applying $16 \mathrm{TEs}$ from $1.176 \mathrm{~ms}$ to $21.816 \mathrm{~ms}$. Starmap ${ }^{\circledR}$ provides a maximum of three successive liver slices showing the spatial distribution of iron. This protocol is routinely used in clinical practice for IOC quantification but clinical interpretation of results is complicated by the use of a scale unit in $\mathrm{Hz} / \mathrm{ms}$. In addition, IOC values $(\mathrm{Hz} / \mathrm{ms})$ are measured from a maximum of 7 ROIs and are presented without standard deviation. An improvement of the Starmap ${ }^{\circledR}$ protocol, called "Advanced Starmap ${ }^{\circledR}$ ", has been developed to express IOC values in $\mu \mathrm{mol} / \mathrm{g}$ ( \pm standard deviation) for each pixel of the liver (Fig. 1C) image. An exponential curve-fitting algorithm $[4,6]$ was applied to determine the T2* value followed by the Wood equation to determine IOC.

For the "Advanced Gandon" and the "Advanced Starmap ${ }^{\circledR}$ ", protocols, the quality of fit $r^{2}$ of the exponential curve is also computed.

\subsection{MRE protocols for viscoelastic assessment}

Magnetic resonance elastography (MRE) experiments have been performed with the MR-Touch ${ }^{\circledR}$ technique composed of a clinical acoustic driver for wave generation at $60 \mathrm{~Hz}$, placed at the same level as the diaphragm and set in contact with the ribcage. The applied frequency was chosen according to the analysis by Leclerc et al. [13] who have characterized the material properties of the acoustic driver. Phase images were recorded in one slice with four temporal offsets, a motion sensitizing gradient echo sequence applied in $\mathrm{Z}$ direction, a flip angle of $30^{\circ}$, a field of view between 36 and $48 \mathrm{~cm}$, a $256 \times 64$ acquisition matrix, a TE/TR equal to $21.7 / 50 \mathrm{~ms}$. Total scan time was $16 \mathrm{~s}$, corresponding to one breath-holding period.

Viscoelastic maps were generated using multi-model direct inversion (MMDI) [14]. For each dataset, phase images were unwrapped using a minimum discontinuity algorithm and filtered with a Butterworth bandpass (from 2 to 128 waves per FOV) to remove longitudinal waves and noise. Assuming the liver is homogeneous, isotropic, and incompressible, the complex shear modulus $\mathrm{G}^{*}=\mathrm{G}^{\prime}+i \mathrm{G}^{\prime \prime}\left(\mathrm{G}^{\prime}\right.$ : storage modulus, $\mathrm{G}^{\prime \prime}$ : loss modulus) (Fig. 2) was calculated with a liver density [14] of $1000 \mathrm{~kg} / \mathrm{m}^{3}$. The mean and the standard deviation of $\mathrm{G}^{\prime}$ and $\mathrm{G}^{\prime \prime}$ were estimated in each ROI.

Moreover, a confidence map [14] is designed to help localize regions of reliable information on the elastogram.

\subsection{Statistical analysis}

The estimated error associated with each method has been evaluated with the coefficient of variation $(\mathrm{CoV})$ used to assess 


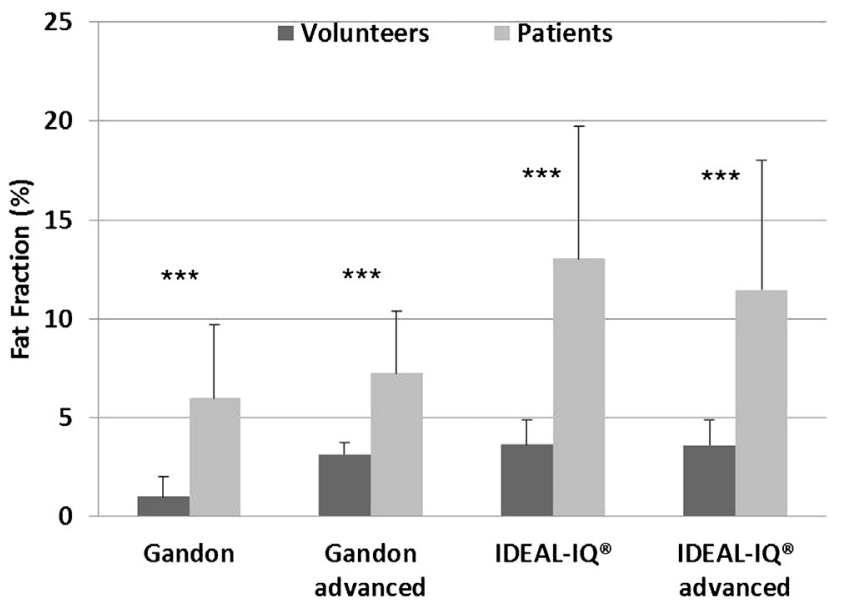

Fig. 3. Fat fraction (median values \pm SD) measured with clinical (IDEAL-IQ ${ }^{\circledR}$ ) and advanced (Gandon, IDEAL-IQ ${ }^{\circledR}$ ) protocols for healthy subjects and patients $\left({ }^{* * *} p<0.001\right)$.

the variability of measurements (iron concentration, fat fraction, viscoelastic properties) for the different protocols. This parameter is calculated as the ratio between the standard deviation of the mean and the mean value across examined subjects.

Two-sample Kolmogorov-Smirnov tests were performed in order to compare the fat fraction data, and the iron concentrations, obtained with both clinical (Gandon, Starmap ${ }^{\circledR}$, IDEAL-IQ ${ }^{\circledR}$ ) and advanced protocols for each cohort (healthy and pathological). The statistical analysis was considered significant for $\mathrm{P}<0.1$ using the software SYSTAT (Systat Software, San Jose, CA).

\section{Results}

The values measured for the different fat protocols (clinical vs advanced), and for the, different iron protocols, were compared from the same three regions of interest (ROIs) which were manually positioned in segments VII (posterior superior area) and VIII (inferior superior area) of the liver. When the FOV or pixel sizes were different between protocols, we co-registered the images by affine transformation in order to compare the same ROIs. Thus, each ROI has the same area and corresponds to 489 pixels in an image of $384 \times 384$ pixels.

\subsection{Comparisons of fat quantification protocols}

Fig. 3 summarized the fat fraction (FF) data provided by the two clinical (Gandon, IDEAL-IQ ${ }^{\circledR}$ ) and their respective advanced protocols. Median values have been presented due to the absence of standard deviation provided by Gandon's protocol. Because IDEAL-IQ ${ }^{\circledR}$ is routinely used in clinical settings to depict the level of FF, this measure was considered as the FF reference for our study. For healthy volunteers, the advanced IDEAL-IQ ${ }^{\circledR}$ and both Gandon protocols confirmed the FF reference result (about $\approx 3 \%$ ), with nearly identical values for no presence of steatosis $(<5 \%)$. For patients, the presence of steatosis was detected by the clinical $(13.0 \pm 6.7 \%)$ and advanced $(11.5 \pm 6.6 \%)$ IDEAL-IQ ${ }^{\circledR}$ protocols, while both Gandon protocols significantly $(p<0.001)$ underestimated the FF

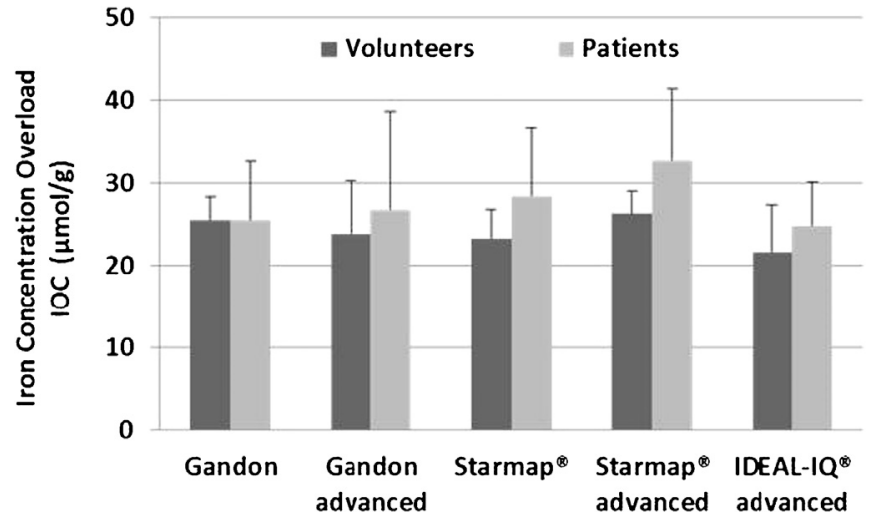

Fig. 4. Iron overload concentration (median values \pm SD) measured with two clinical (Gandon, Starmap ${ }^{\circledR}$ ) protocols and three advanced (Gandon, Starmap ${ }^{\circledR}$, IDEAL-IQ $\left.{ }^{\circledR}\right)$ iron protocols for healthy subjects and patients.

value (about $\approx 7 \%$ ) compared to IDEAL-IQ ${ }^{\circledR}$. The advanced Gandon protocol revealed the lower coefficient of variation $(\mathrm{CoV}=31 \%)$ compared to the other protocols $\left(\mathrm{CoV} \_\right.$advanced IDEAL-IQ $=33.2 \%$, CoV_IDEAL-IQ $\left.{ }^{\circledR}=35.6 \%\right)$. The performance of the four protocols as a means of diagnosing fat fraction was validated by the capability to significantly differentiate $(p<0.001)$ healthy and steatosis livers.

\subsection{Comparisons of iron overload quantification protocols}

Fig. 4 summarizes the results of iron overload concentration (IOC) obtained with the clinical (Gandon, Starmap ${ }^{\circledR}$ ) and advanced protocols (Gandon, Starmap ${ }^{\circledR}$, IDEAL-IQ ${ }^{\circledR}$ ). IOC data are shown at the median value due to the absence of intra subject standard deviation provided by Starmap ${ }^{\circledR}$ and considered as the reference data in our study. Note that the standard deviation of each IOC value measured with Gandon protocol is not known exactly; no $\mathrm{SD}$ is available from R2* acquisitions with IDEAL-IQ ${ }^{\circledR}$. Thus, the Starmap ${ }^{\circledR}$ median values for healthy and patients are $23.3 \pm 3.6 \mu \mathrm{mol} / \mathrm{g}$ and $28.5 \pm 8.2 \mu \mathrm{mol} / \mathrm{g}$, respectively. These results confirm a normal level $(<36 \mu \mathrm{mol} / \mathrm{g})$ of iron for both healthy subjects and fibrosis patients.

IOC values captured for healthy livers using the clinical protocols (Starmap ${ }^{\circledR}$, Gandon) are in the same range as those captured using the advanced iron protocols (Starmap ${ }^{\circledR}$, Gandon, IDEAL-IQ $^{\circledR}$ ). Fibrosis patients were correctly diagnosed with no hemochromatosis with both Starmap ${ }^{\circledR}(28.5 \pm 8.2 \mu \mathrm{mol} / \mathrm{g}$ and $32.7 \pm 8.7 \mu \mathrm{mol} / \mathrm{g}$ ) protocols and with both Gandon (25.5 $\pm 2.8 \mu \mathrm{mol} / \mathrm{g}$ and $23.9 \pm 6.4 \mu \mathrm{mol} / \mathrm{g}$ ) protocols. A higher quality $\left(r^{2}\right)$ of fit was found with the advanced Starmap ${ }^{\circledR}$ protocol compared to the advanced Gandon. The coefficient of variation measured for patients is lower (11.5\%) with the advanced Starmap ${ }^{\circledR}$ protocol compared to the other protocols (advanced Gandon: $13.3 \%$, IDEAL-IQ $\left.{ }^{\circledR}: 15.6 \%\right)$. It can be noted that the IDEAL-IQ ${ }^{\circledR}$ protocol underestimated IOC values $(24.8 \pm$ $5.4 \mu \mathrm{mol} / \mathrm{g}$ ) but that patients were correctly diagnosed.

\subsection{Comparisons of viscoelastic measurements}

Fig. 5 shows the median of the storage $\left(\mathrm{G}^{\prime}\right)$ and loss $\left(\mathrm{G}^{\prime \prime}\right)$ moduli measured from the same three ROIs of IOC and FF. 


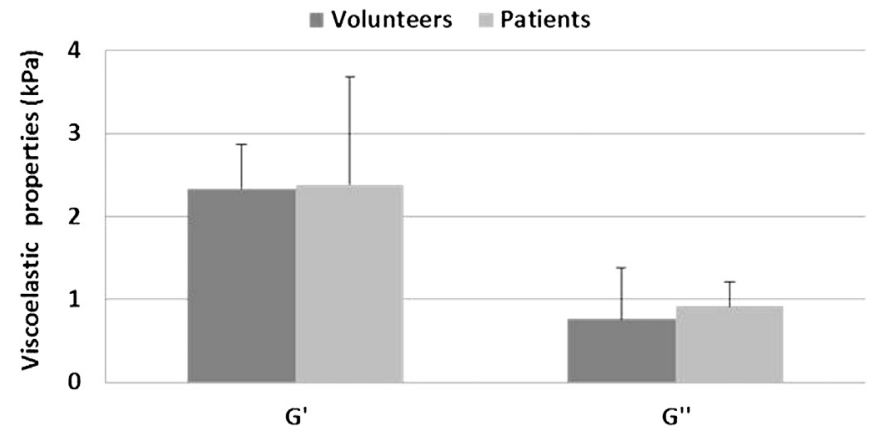

Fig. 5. Median values $( \pm \mathrm{SD})$ of the storage $\left(\mathrm{G}^{\prime}\right)$ and loss $\left(\mathrm{G}^{\prime \prime}\right)$ moduli for healthy volunteers and fibrosis patients.

Both parameters show the same range of data for both cohorts. The median values of $\mathrm{G}^{\prime}$ for healthy and fibrosis patients are $2.33 \pm 0.54 \mathrm{kPa}$ and $2.39 \pm 1.30 \mathrm{kPa}$, respectively. The median values of $\mathrm{G}^{\prime \prime}$ for healthy and patients are $0.76 \pm 0.62 \mathrm{kPa}$ and $0.92 \pm 0.28 \mathrm{kPa}$, respectively.

\section{Discussion}

Many different methods are available to determine steatosis and hemochromatosis and most of the time clinicians use a protocol provided by the MR imaging company. The originality of the present study has been to demonstrate that manufacturer's tools can be improved to simultaneously quantify the fat fraction $(\mathrm{FF})$ and the iron overload concentration (IOC) to save time for the patient and the clinical setting.

Concerning fat fraction measurements, the present study has demonstrated the limits of the clinical Gandon protocol to estimate a normal level of steatosis. In addition, the underestimation of FF values, for patients, provided by both Gandon protocols compared to the IDEAL-IQ ${ }^{\circledR}$ protocols may be due to the measurement of the mean ROI which is made before the application of the exponential fit. This can lead to a mismeasurement of FF when the difference of the IP and OP signal is low. An improvement of the IDEAL-IQ ${ }^{\circledR}$ protocol has allowed an increase in the number of ROIs which are limited to three in the clinical IDEAL-IQ ${ }^{\circledR}$ tool. Furthermore, the advanced protocol is now able to provide instantaneous volume FF, enabling analysis of liver homogeneity along the entire hepatic gland.

For iron quantification, the healthy volunteers have IOC values in agreement with the healthy cut-off $(<36 \mu \mathrm{mol} / \mathrm{g})$ found in the literature $[3,4]$. This result validates the advanced iron protocols (Gandon, Starmap ${ }^{\circledR}$, IDEAL-IQ ${ }^{\circledR}$ ) that were developed in this work.

The improved protocols of Gandon and Starmap ${ }^{\circledR}$ use the same process of exponential fit and the same equation (Wood) to estimate IOC values. Advanced Starmap ${ }^{\circledR}$ seems more accurate for measuring IOC compared to the advanced Gandon. This can be explained by the fact that the MR sequence in Starmap protocol uses 16 TEs compared to 8 in Gandon's protocol. In addition, the advanced Gandon protocol accounts for background noise, thus providing higher IOC values than Starmap ${ }^{\circledR}$ which has no background subtraction [16]. Moreover, the first TE in the Advanced Starmap ${ }^{\circledR}$ protocol is lower $(1.176 \mathrm{~ms})$ than in Advanced Gandon ( $2.4 \mathrm{~ms}$ ) leading to the acquisition of more GRE MR signal which is usually low in the case of iron overload [17].

The Gandon protocol used in clinical practice is not appropriate to detect lower stages of hemochromatosis due to its high standard deviation (about $20 \mu \mathrm{mol} / \mathrm{g}$ ), as also found in other studies [3-5]. The improvement of Gandon's method now allows the assessment of low-level hemochromatosis as well as the interpretation of IOC through cartography.

The present work has demonstrated that IOC values can also be measured from MRI protocols that are otherwise dedicated to fat fraction estimates (IDEAL-IQ ${ }^{\circledR}$ ). In clinical practice, the $\mathrm{Hz} / \mathrm{ms}$ unit used for R2* acquisitions complicates qualitatively assessments of IOC level [18]. An improved version of this protocol has demonstrated the ability to provide 32 iron cartographies with quantitative IOC values $(\mu \mathrm{mol} / \mathrm{g} \pm$ standard deviation) from unlimited ROIs.

In parallel to MRI protocols, others noninvasive imaging tools such as ultrasound elastography $[19,20]$ (Fibroscan) and magnetic resonance elastography (MRE) $[15,17,22]$ have demonstrated their ability to evaluate steatosis. Indeed, Fibroscan can provide an estimate of steatosis through the measurement of a novel parameter called CAP (control attenuation parameter) $[19,23]$. Moreover, the magnetic resonance elastography (MRE) technique is also able to assess steatosis through measurement of the storage modulus [24]. In this work, the fibrosis patients have viscoelastic data in agreement with results found in the literature [21,25] for healthy liver. This result is not surprising due to the low level of fibrosis within the patients. In perspective, the advanced Gandon, Starmap and IDEAL-IQ ${ }^{\circledR}$ protocols could be applied to a larger cohort of patients and correlation made with functional information obtained by elastographic measurements.

A phantom study (with variable iron and fat content) could be prepared for the validation of the advanced protocols. In addition, future studies could test MR Spin Echo sequence instead of MR GRE sequence to diagnose high level of iron overload, and to deduce R2 map of liver on the basis of St Pierre's study [19] (Ferriscan ${ }^{\circledR}$ protocol). The main advantage of this protocol is that the measurement technique has been calibrated and validated against biopsy (i) across multiple scanners, (ii) in patients with different stages of fibrosis, and (iii) in both pediatrics and adult.

To conclude, the improvements of liver MR imaging clinical protocols to simultaneously quantify iron overload, steatosis and stiffness will contribute to make MRI become a primary modality for liver analysis. It may help to replace the liver biopsy with its inherent risk, invasiveness, and sampling error.

\section{Conflict of interest statement}

The authors have declared that no competing interests exist.

\section{Financial support}

This study was funded by Echosens company. 


\section{Acknowledgements}

Contributions: Study concept and design, acquisition of data, analysis and interpretation of data, drafting of the manuscript and critical revision of the manuscript: Redouane Ternifi, Philippe Pouletaut, Magali Sasso, Véronique Miette, Fabrice Charleux, Sabine F. Bensamoun.

\section{References}

[1] Friedman SL. Mechanisms of hepatic fibrogenesis. Gastroenterology 2008;134(6):1655-69.

[2] Reeder SB, Sirlin CB. Quantification of liver fat with magnetic resonance imaging. Magn Reson Imaging Clin N Am 2010;18:337-57.

[3] Gandon Y, Olivié D, Guyader D, et al. Non-invasive assessment of hepatic iron stores by MRI. Lancet 2004;363:357-62.

[4] Alústiza Echeverría JM, Castiella A, Emparanza JI. Quantification of iron concentration in the liver by MRI. Insights Imaging 2012;3:173-80.

[5] Boulic Y, Kiani A, Bardou-Jacquet A, et al. Abstract Archives of the RSNA, Radiological Society of North America; 2013.

[6] Wood JC, Enriquez C, Ghugre N, et al. MRI R2 and R2* mapping accurately estimates hepatic iron concentration in transfusion-dependent thalassemia and sickle cell disease patients. Blood 2005;106:1460-5.

[7] Reeder SB, Robson PM, Yu H, et al. Quantification of hepatic steatosis with MRI: the effects of accurate fat spectral modeling. J Magn Reson Imaging 2009;29:1332-9.

[8] Kuhn JP, Hernando D, Munoz del Rio A, et al. Effect of multipeak spectral modeling of fat for liver iron and fat quantification: correlation of biopsy with MR imaging results. Radiology 2012;265:133-42.

[9] Schneider CA, Rasband WS, Eliceiri KW. NIH Image to ImageJ: 25 years of image analysis. Nat Methods 2012;9:671-5.

[10] Gandon Y. IronByMR. Available from: https://imagemed.univ-rennes1.fr/ ironbymr.

[11] Glover GH, Schneider E. Three-point Dixon technique for true water/fat decomposition with B0 inhomogeneity correction. Magn Reson Med 1991;18:371-83.

[12] Guiu B, Loffroy R, Ben Salem D, et al. Liver steatosis and in-out of phase MR imaging: theory and clinical applications at 3T. J Radiol 2007;88:1845-53.
[13] Leclerc GE, Debernard L, Foucart F, et al. Characterization of a hyperviscoelastic phantom mimicking biological soft tissue using an abdominal pneumatic driver with magnetic resonance elastography (MRE). J Biomech 2012;45:952-7.

[14] Silva AM, Grimm RC, Glaser KJ, et al. Magnetic resonance elastography: evaluation of new inversion algorithm and quantitative analysis method. Abdom Imaging 2015;40:810-7.

[15] Leclerc GE, Charleux F, Robert L, et al. Analysis of liver viscosity behavior as a function of multifrequency magnetic resonance elastography (MMRE) postprocessing. J Magn Reson Imaging 2013;38:422-8.

[16] Ghugre NR, Enriquez CM, Coates TD, et al. Improved R2* measurements in myocardial iron overload. J Magn Reson Imaging 2006;23:9-16.

[17] Mariappan YK, Dzyubak B, Glaser KJ, et al. Application of modified spin-echo-based sequences for hepatic MR elastography: evaluation, comparison with the conventional gradient-echo sequence, and preliminary clinical experience. Radiology 2016;282:390-8.

[18] St Pierre TG, El-Beshlawy A, Elalfy M, et al. Multicenter validation of spin-density projection-assisted R2-MRI for the noninvasive measurement of liver iron concentration. Magn Reson Med Jun 2014;71(6):2215-23.

[19] Nguyen-Khac E, Chatelain D, Tramier B, et al. Assessment of asymptomatic liver fibrosis in alcoholic patients using fibroscan: prospective comparison with seven non-invasive laboratory tests. Aliment Pharmacol Ther 2008;28:1188-98.

[20] Myers RP, Pollett A, Kirsch R, et al. Controlled Attenuation Parameter (CAP): a noninvasive method for the detection of hepatic steatosis based on transient elastography. Liver 2012;32:902-10.

[21] Bensamoun SF, Wang L, Robert L, et al. Measurement of liver stiffness with two imaging techniques: magnetic resonance elastography and ultrasound elastometry. J Magn Reson Imaging 2008;28:1287-92.

[22] Bensamoun SF, Leclerc GE, Debernard L, et al. Cutoff values for alcoholic liver fibrosis using magnetic resonance elastography technique. Alcohol Clin Exp Res 2013;37:811-7.

[23] Sasso M, Audière S, Kemgang A, et al. Liver steatosis assessed by controlled attenuation parameter (CAP) measured with the XL probe of the FibroScan: a pilot study assessing diagnostic accuracy. Ultrasound Med Biol 2016;42:92-103.

[24] Chen J, Talwalkar JA, Yin M, et al. Early detection of nonalcoholic steatohepatitis in patients with nonalcoholic fatty liver disease by using MR elastography. Radiology 2011;259:749-56.

[25] Venkatesh SK, Ehman RL. Magnetic resonance elastography of liver. Magn Reson Imaging Clin N Am 2014;22:433-46. 